\title{
A ketogenic diet suppresses seizures in mice through adenosine $A_{1}$ receptors
}

\author{
Susan A. Masino, ${ }^{1}$ Tianfu Li, ${ }^{2}$ Panos Theofilas, ${ }^{2}$ Ursula S. Sandau, ${ }^{2}$ David N. Ruskin, ${ }^{1}$ \\ Bertil B. Fredholm, ${ }^{3}$ Jonathan D. Geiger, ${ }^{4}$ Eleonora Aronica, ${ }^{5}$ and Detlev Boison ${ }^{2}$

\begin{abstract}
1Department of Psychology and Neuroscience Program, Trinity College, Hartford, Connecticut, USA. 2R.S. Dow Neurobiology Laboratories, Legacy Research Institute, Portland, Oregon, USA. ${ }^{3}$ Department of Physiology and Pharmacology, Karolinska Institutet, Stockholm, Sweden. ${ }^{4}$ Department of Pharmacology, Physiology, and Therapeutics, University of North Dakota, Grand Forks, North Dakota, USA. 5Department of Neuropathology, University of Amsterdam, Amsterdam, The Netherlands.
\end{abstract}

\begin{abstract}
A ketogenic diet (KD) is a high-fat, low-carbohydrate metabolic regimen; its effectiveness in the treatment of refractory epilepsy suggests that the mechanisms underlying its anticonvulsive effects differ from those targeted by conventional antiepileptic drugs. Recently, KD and analogous metabolic strategies have shown therapeutic promise in other neurologic disorders, such as reducing brain injury, pain, and inflammation. Here, we have shown that KD can reduce seizures in mice by increasing activation of adenosine $A_{1}$ receptors $\left(A_{1} R s\right)$. When transgenic mice with spontaneous seizures caused by deficiency in adenosine metabolism or signaling were fed $\mathrm{KD}$, seizures were nearly abolished if mice had intact $A_{1} R s$, were reduced if mice expressed reduced $A_{1} R s$, and were unaltered if mice lacked $A_{1} R s$. Seizures were restored by injecting either glucose (metabolic reversal) or an $A_{1} R$ antagonist (pharmacologic reversal). Western blot analysis demonstrated that the KD reduced adenosine kinase, the major adenosine-metabolizing enzyme. Importantly, hippocampal tissue resected from patients with medically intractable epilepsy demonstrated increased adenosine kinase. We therefore conclude that adenosine deficiency may be relevant to human epilepsy and that KD can reduce seizures by increasing $\mathrm{A}_{1} \mathrm{R}$-mediated inhibition.
\end{abstract}

\section{Introduction}

A ketogenic diet (KD) is an alternative metabolic treatment for epilepsy, and multiple retrospective and prospective studies confirm its clinical benefits $(1,2)$. The high-fat, low-carbohydrate composition of a KD forces ketone- rather than glucose-based metabolism, but it is not known how this shift leads to anticonvulsant consequences. Primary applications of KD therapy include pediatric and medically refractory epilepsy; its use is increasing globally, and clinical benefits are similar across cultures and age groups $(1,2)$. Despite its success, side effects and requisite strict compliance have limited widespread use of KD, and a diet-based approach is often considered as a last resort. Better understanding of the mechanisms involved in the anticonvulsant actions of a KD could lead to development of pharmacological strategies that take advantage of beneficial aspects and limit problems associated with diet therapy.

Adenosine acting at adenosine $A_{1}$ receptors $\left(A_{1} R s\right)$ is a logical candidate for the effects of KD therapy (3). Adenosine is well-established as a powerful anticonvulsant (4), and endogenous adenosine acting at $A_{1} R s$ is an important seizure-control mechanism (5). Moreover, deletion of $A_{1}$ Rs and increased adenosine clearance by elevated adenosine kinase (ADK) both cause spontaneous intrahippocampal electrographic seizures (6) and increase the brain's susceptibility to injury (7). Conversely, therapeutic adenosine augmentation is highly effective in controlling seizures (8). Adenosine is the core of ATP, a key molecule in basic biochemistry and a ligand at its own family of $\mathrm{G}$ protein-coupled cell surface receptors. Thus, adenosine is a homeostatic bioenergetic network regulator involved in metabolism and ongoing neuronal activity and is well-positioned

Conflict of interest: The authors have declared that no conflict of interest exists. Citation for this article: JClin Invest. 2011;121(7):2679-2683. doi:10.1172/JCI57813. to translate metabolic changes into altered brain activity. Using 3 lines of transgenic mice that all exhibit electrographic seizures as a result of deficient adenosine signaling (6), we present here what we believe to be the first direct evidence that adenosine acting at $A_{1} R s$ contributes to the therapeutic effects of KDs.

\section{Results and Discussion}

$A_{1} R$ activation suppresses seizures. In line with our previous demonstration (6), $A_{1} R^{+/-}$mice, $A_{1} R^{-/-}$mice, and $A d k-T g$ mice (based on expression of a ubiquitously expressed $A d k$ transgene on an Adknull background) maintained on a control diet (CD) experienced regular electrographic hippocampal seizures consistent with deficient adenosine $/ A_{1} R$ signaling (Figure 1 ). Seizure frequency and duration were similar between $A d k-T g$ and $A_{1} R^{-/-}$mice and significantly lower in $A_{1} R^{+/-}$mice (but still elevated above WT; Table 1). Behavioral (clinical) seizures were never observed in the mutants, and electrographic seizures were never observed in the WT group.

To quantify the effects of KD, WT and transgenic mice were fed CD or KD. At 3 weeks, KD nearly abolished seizures in Adk-Tg mice and significantly reduced the duration of remaining seizures (Table 1 and Figure 1). If KD suppresses seizures in $A d k-T g$ mice by elevating adenosine in brain, we predicted that its seizure-suppressing potential would be reduced in $A_{1} R^{+/-}$mice, which have only $50 \%$ of this receptor and lowered sensitivity to adenosine (9). As expected, KD-fed $A_{1} R^{+/-}$mice experienced a significant, but lesser (approximately 50\%), reduction in seizure frequency, although seizure duration was not changed compared with CD-fed $A_{1} R^{+/-}$mice (Table 1 ). We further predicted that spontaneous seizures in $A_{1} R^{-/-}$mice would be resistant to the beneficial effects of $\mathrm{KD}$ if its protective mechanism involves activation of $\mathrm{A}_{1}$ Rs. Indeed, $\mathrm{KD}$ was completely ineffective in affecting seizures in $A_{1} R^{-/-}$mice. These findings demonstrate 


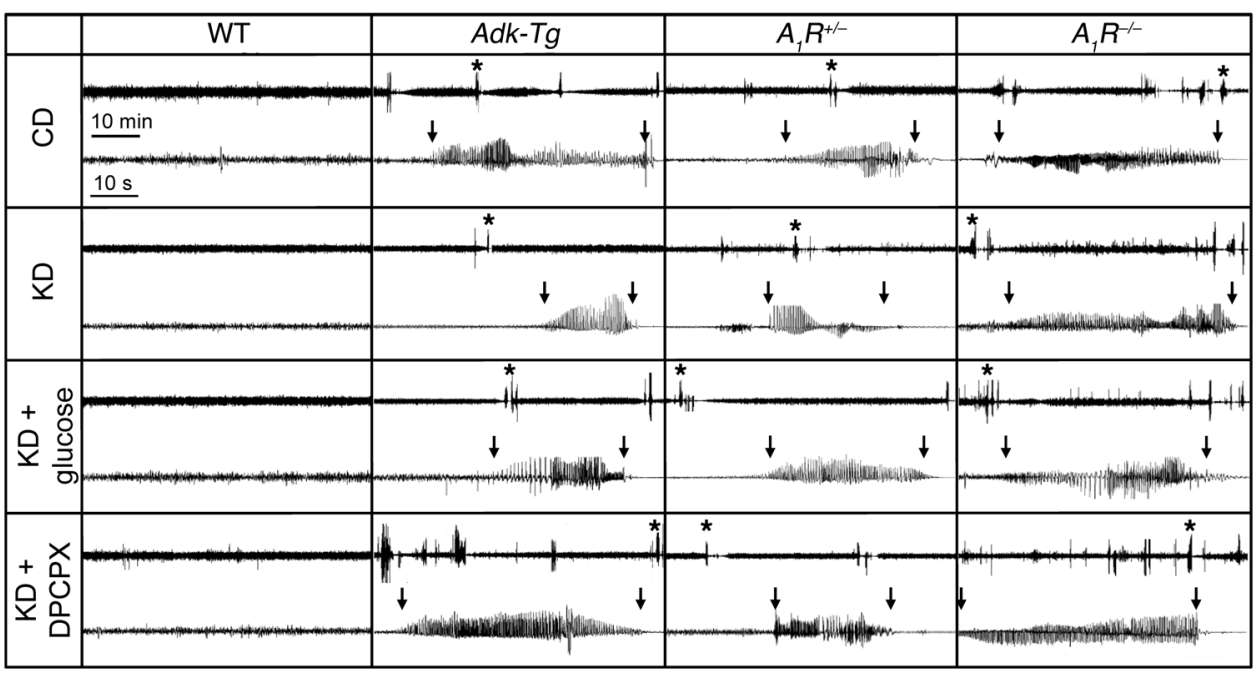

Figure 1

Seizure suppression by $K D$ depends on $A_{1} R$ activation. Representative $E E G$ recordings from the $C A 3$ of WT and transgenic mice reflect seizure distribution over a 1-hour time span (top traces) and individual seizures at higher resolution (1 minute; bottom traces). Asterisks in top traces denote the individual seizures chosen. Beginning and end of seizures are marked by vertical arrows. Traces from CD-fed animals showed baseline seizure activity in all mutants and lack of seizures in WT. KD almost completely abolished seizures in Adk-Tg mice; rare seizures were of reduced duration, as shown. KD reduced seizure activity in $A_{1} R^{+/-}$mice, had no effect in $A_{1} R^{-/-}$mice. Treatment with glucose or DPCPX reversed $\mathrm{KD}$ effects. See Table 1 for quantitation.

that KD suppresses seizures caused by adenosine deficiency (Adk-Tg) or reduced adenosine signaling $\left(A_{1} R^{+/-}\right)$, but has no effect in the absence of $\mathrm{A}_{1} \mathrm{Rs}\left(A_{1} R^{-/-}\right)$.

Seizures are restored with glucose or $A_{1} R$ blocker treatment. To determine the extent to which the reduced frequency of spontaneous seizures in $A d k-T g$ and $A_{1} R^{+/-}$mice was specifically the result of the low-carbohydrate nature of KD, we injected glucose into KD-fed mice of each genotype. In $A d k-T g$ and $A_{1} R^{+/-}$mice, which displayed reduced seizures after $\mathrm{KD}$, glucose injection increased seizure frequency significantly within 30-90 minutes; in Adk-Tg mice, the increase did not quite reestablish the baseline CD-fed seizure phenotype (Table 1 and Figure 1).

The loss of KD-induced seizure suppression in $A_{1} R^{-/-}$mice, combined with the reduced efficacy of KD in $A_{1} R^{+/-}$compared with $A d k-T g$ mice, is indicative of seizure suppression via a mechanism involving $A_{1}$ Rs. To test this further, we injected a nonconvulsive dose of 8-cyclopentyl-1,3-dipropylxanthine (DPCPX; $1 \mathrm{mg} / \mathrm{kg}$ ), an $\mathrm{A}_{1}$ R-selective antagonist. In $A d k-T g$ and $A_{1} R^{+/-}$mice, DPCPX restored seizure frequency to control levels, whereas it had no significant effect in WT or $A_{1} R^{-/-}$mice (Figure 1 and Table 1). DPCPX in KD-fed $A d k-T g$ mice increased seizure duration significantly compared with both $\mathrm{CD}$ and $\mathrm{KD}$ conditions; however, it did not change seizure duration in $A_{1} R^{+/-}$mice, which suggests that its effect on duration depended on the relative abundance of $\mathrm{A}_{1}$ Rs. As expected, DPCPX had no effect on seizures in $A_{1} R^{-/-}$mice. These data, coupled with the lack of KD efficacy in $A_{1} R^{-/-}$mice, demonstrated that $\mathrm{A}_{1}$ Rs are a molecular target whereby KD reduces ictogenesis in vivo.

WT and $A_{1} R^{-1-}$ mice have similar metabolic responses. To confirm that $A_{1} R^{-/-}$mice experienced a similar metabolic response to $\mathrm{KD}$, we measured the plasma level of $\beta$-hydroxybutyrate $(\beta \mathrm{HB})$ in $A_{1} R^{-/}$ involved in an endogenous protective mechanism of the brain in response to stress or injury (10). We therefore hypothesized that metabolic stress imposed by KD might likewise lead to reduction in brain ADK. KD- or CD-fed WT mice were sacrificed at 3 and 4 weeks of feeding, corresponding to the time span of seizure analysis above. Western blot showed that this key adenosine-regulating enzyme was downregulated significantly in the KD group (Figure 2), which suggests a possible mechanism for KD's seizure suppression.

\section{Table 1}

Seizure frequency and duration

\begin{tabular}{|c|c|c|c|c|}
\hline & CD & KD & $\begin{array}{l}\text { KD plus } \\
\text { glucose }\end{array}$ & $\begin{array}{l}\text { KD plus } \\
\text { DPCPX }\end{array}$ \\
\hline \multicolumn{5}{|c|}{ Frequency (seizures/h) } \\
\hline $\begin{array}{l}\text { WT } \\
\text { Adk-Tg } \\
A_{1} R^{+-} \\
A_{1} R^{--}\end{array}$ & $\begin{array}{c}0 \\
4.8 \pm 0.4 \\
3.8 \pm 0.7 \\
5.3 \pm 0.6\end{array}$ & $\begin{array}{c}0 \\
0.6 \pm 0.2^{\mathrm{A}} \\
1.8 \pm 0.4^{\mathrm{A}} \\
5.1 \pm 0.6\end{array}$ & \begin{tabular}{l}
\multicolumn{1}{c}{0} \\
$4.1 \pm 0.2^{\mathrm{B}, \mathrm{C}}$ \\
$3.4 \pm 0.3^{\mathrm{C}}$ \\
$5.2 \pm 0.5$
\end{tabular} & $\begin{array}{c}0 \\
4.9 \pm 0.5^{c} \\
3.9 \pm 0.7^{c} \\
4.9 \pm 0.5\end{array}$ \\
\hline \multicolumn{5}{|c|}{ Duration (s) } \\
\hline $\begin{array}{l}\text { WT } \\
\text { Adk-Tg } \\
A_{1} R^{+-} \\
A_{1} R^{--}\end{array}$ & $\begin{array}{c}0 \\
26.8 \pm 3.3 \\
16.4 \pm 1.6 \\
27.9 \pm 2.4\end{array}$ & $\begin{array}{c}0 \\
14.4 \pm 4.0^{\mathrm{A}} \\
15.9 \pm 4.2 \\
29.3 \pm 2.9\end{array}$ & $\begin{array}{c}0 \\
18.6 \pm 2.0^{\mathrm{B}, \mathrm{D}} \\
16.2 \pm 2.1 \\
31.2 \pm 4.9\end{array}$ & $\begin{array}{c}0 \\
37.6 \pm 4.3^{\mathrm{A}, \mathrm{C}} \\
16.4 \pm 2.2 \\
29.9 \pm 3.1\end{array}$ \\
\hline
\end{tabular}

Mice were maintained on $\mathrm{CD}$ or $\mathrm{KD}$ for 3 weeks. KD reduced spontaneous seizures in Adk-Tg and $A_{1} R^{+/-}$mice and was ineffective in $A_{1} R^{-1-}$ mice; glucose (2 g/kg i.p.) or DPCPX (1 $\mathrm{mg} / \mathrm{kg}$ i.p.) reversed its anti-ictogenic effects. Values are mean \pm SD. $n=10(\mathrm{CD}, \mathrm{KD}) ; 5$ (KD plus glucose, KD plus DPCPX). ${ }^{A} P<0.001,{ }^{B} P<0.05$ vs. $C D$.

$\mathrm{C} P<0.001$, $\mathrm{D} P<0.05$ vs. KD alone. 

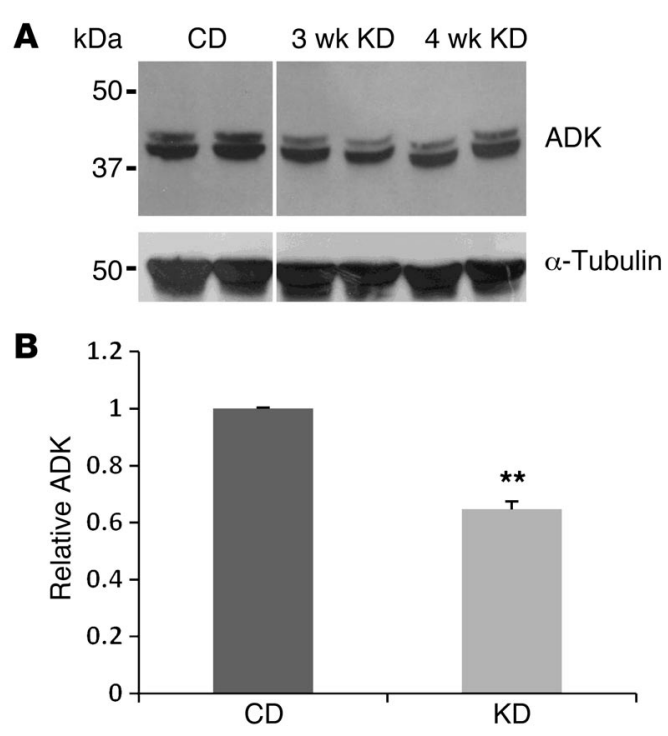

ADK overexpression in buman epilepsy. We have demonstrated previously that astrogliosis, ADK overexpression, and resulting adenosine deficiency are implicated in seizure generation in rodent models of epilepsy $(6,10)$. Therefore, reconstitution of normal adenosine signaling by KD might be of therapeutic value for epileptic patients with refractory temporal lobe epilepsy (TLE). To investigate whether adenosine dysfunction is likewise implicated in the pathology of human epilepsy, we studied the expression of ADK in hippocampus of patients with hippocampal sclerosis (HS) and histologically normal human hippocampus. ADK was upregulated within specific hippocampal regions in HS specimens compared with control hippocampus (Figure 3, A-D). Abundant ADK-immunopositive cells with typical astroglial morphology were observed in areas with prominent gliosis in all HS specimens; double-labeling confirmed ADK expression in glial fibrillary acidic protein-positive (GFAP-positive) reactive astrocytes (Figure 3, $\mathrm{B}$ and D). Western blot analysis confirmed a greater than 3-fold increase in ADK levels in hippocampus of TLE patients (Figure 3, $\mathrm{E}$ and $\mathrm{F}$ ), which suggests that adenosine deficiency could contribute to the epileptic phenotype in those patients.

Using transgenic mice and complementary pharmacology, we demonstrated that KD-induced seizure control was dependent on $\mathrm{A}_{1}$ Rs: KD virtually abolished seizures in Adk-Tg mice (with reduced endogenous adenosine and intact $\mathrm{A}_{1} \mathrm{Rs}$ ), reduced seizures partially but significantly in $A_{1} R^{+/-}$mice, and had no effects in $A_{1} R^{-/-}$mice or in the presence of $A_{1} R$ blockade. We showed overexpression of $\mathrm{ADK}$ (which should reduce $\mathrm{A}_{1} \mathrm{R}$ activation) in human epilepsy and $\mathrm{KD}$-induced reduction of $\mathrm{ADK}$ in mouse brain, thus providing a mechanism for KD-induced increase in adenosine and anticonvulsant effects of $K D$ via $A_{1}$ Rs. Whereas altered adenosinergic signaling might not be the underlying cause for seizures in all epilepsies, adenosine has stopped seizures in every seizure model tested to date, including models of pharmacoresistant epilepsy. Thus, no matter the cause of the seizure, adenosine can help to resolve it, provided - as shown here - that $\mathrm{A}_{1}$ Rs are present. Subclinical, brief electrographic seizure events (undetectable with scalp electrodes), as exhibited in all 3 strains of transgenic mice in the present study, are now increasingly recognized and likely play an important role in early epileptogenesis (11).

\section{Figure 2}

KD leads to downregulation of ADK. (A) Representative Western blot from brain extracts of WT mice fed CD or KD for 3 and 4 weeks. Note the 2 different splice variants of ADK in the ADK-reactive bands. Antitubulin immunoreactivity was used to normalize for equal loading. Lanes were run on the same gel but were noncontiguous (white line). (B) Brain ADK from mice fed CD or KD for 3-4 weeks, expressed relative to $C D$ ( $n=4$ per group). Data are mean \pm SEM. ${ }^{* *} P<0.01$ vs. CD.

Acute reversal of seizure suppression by glucose confirmed the specificity of KD's metabolic effects and comported with clinical observations and animal studies highlighting the importance of low glucose to the effects of KD (12). Indeed, we recently demonstrated in vitro that metabolic consequences of $\mathrm{KD}$ - reduced extracellular glucose and increased intracellular ATP - produce $A_{1}$ R-dependent inhibition of cornu ammonis region 3 (CA3) pyramidal cells (3). Of interest, glucose-induced seizure reversal in $A d k-T g$ mice was not complete (which indicates that the specific enzymatic activity of ADK might have been increased based on rapid glucose-dependent restoration of the energy charge), although ADK levels were still quantitatively reduced (Figure 2). Additional mechanisms might also contribute to KD-induced seizure suppression: in vitro studies demonstrated direct acute effects of ketones $(13,14)$, although the relative importance of these mechanisms to the chronic in vivo effects of KD administration in humans remains to be determined.

When relating these findings to human epilepsy, we found that brain tissue resected from humans with intractable epilepsy showed increased ADK - and therefore likely a relative adenosine deficiency. Previously, adenosine deficiency has been demonstrated directly in microdialysis samples from epileptogenic hippocampus in human patients with TLE (15). These results and our current data indicate that alteration of adenosine signaling is relevant to human epilepsy. Specifically, at least some forms of human epilepsy replicate key features of our adenosine-deficient model, increasing the translational potential of these results. Together, these data delineate a clinically relevant relationship among KD, adenosine, and epilepsy, which could lead to less-restrictive diets, alternate pharmaceutical approaches, and broader applications of metabolic strategies to different medical conditions.

\section{Methods}

Animals. All animal procedures were approved by the Legacy Institutional Animal Care and Use Committee. Adk-Tg mice are based on expression of a ubiquitously expressed $A d k$ transgene on an $A d k$-null background, leading to brain-wide $\mathrm{ADK}$ overexpression (6). $A_{1} R^{+/-}$and $A_{1} R^{-/-}$mice lack 1 or both $A_{1} R$ alleles (9). All animals were maintained on a C57BL/ 6 background. Male mice were fed either CD or KD (product no. F3666; Bioserv) ad libitum for 4 weeks ( $n=10$ per genotype). To reverse KD effects, animals received i.p. injection of either glucose in water $(30 \% \mathrm{w} / \mathrm{v} ; 2 \mathrm{~g} / \mathrm{kg})$ or DPCPX $(1 \mathrm{mg} / \mathrm{kg} ; 2 \mathrm{ml} / \mathrm{kg}$ in $20 \%$ DMSO [w/v]).

EEG studies. Bipolar, coated stainless-steel electrodes were implanted into the right $\mathrm{CA} 3$, and monopolar reference and ground electrodes were affixed above cortex and cerebellum, as described previously (6). 24 hours after implantation, we recorded 12 hours of continuous EEG from each animal using a Nervus EEG recording system (6). Seizures were defined as high-amplitude rhythmic discharges (repetitive spikes, spike-and-wave discharges, slow waves) lasting at least 5 seconds. After 12 hours, each animal received glucose or DPCPX, and EEG monitoring resumed for another 12 hours. All EEG recordings were performed at 3-4 weeks of diet treatment and within 48 hours of electrode implantation to exclude chronic reactions to the electrodes. 
A

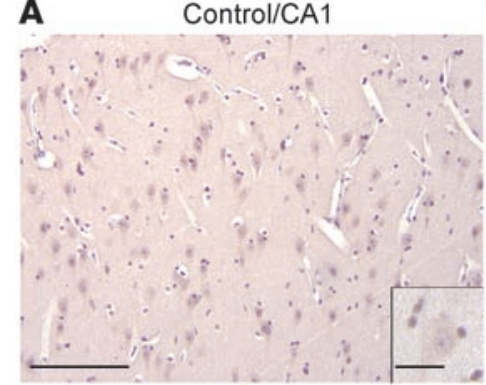

C

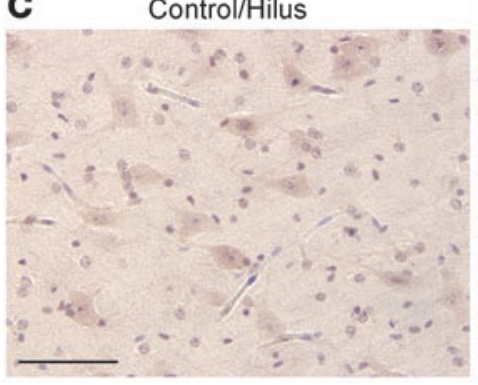

B

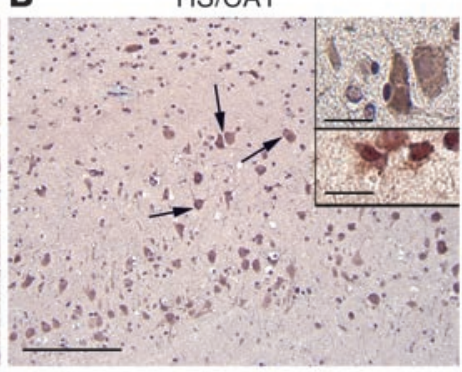

D

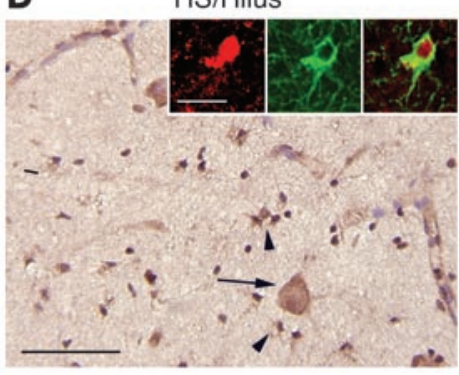

E

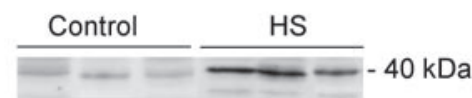

$\mathbf{F}$

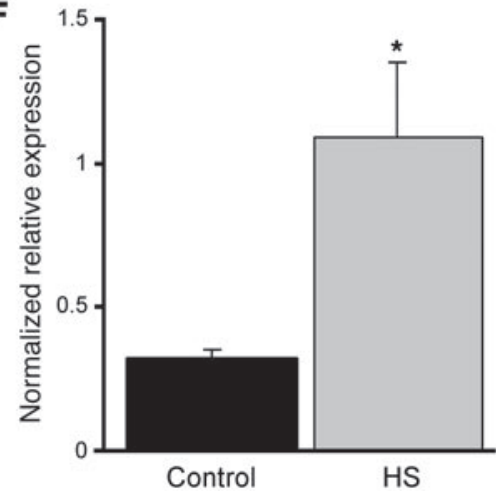

\section{Figure 3}

ADK immunoreactivity in hippocampus of control and TLE patients with medial temporal HS. (A-D) Sections were counterstained with hematoxylin. Shown are representative CA1 (A and B) and hilus (C and D) from the same sample. (A and C) Control hippocampus showed weak ADK immunoreactivity. Histologically normal surgical hippocampus displayed an immunoreactivity pattern similar to that in control autopsy hippocampus (not shown). (B and $\mathbf{D}$ ) The HS specimen demonstrated increased ADK expression in both residual pyramidal and hilar neurons (arrows and $\mathbf{B}$, top inset) and in reactive astrocytes (arrowheads and $\mathbf{B}$, bottom inset). Insets in $\mathbf{D}$ show expression of ADK (red) in a reactive astrocyte (GFAP, green). Scale bars: $160 \mu \mathrm{m}$ (A and B); $80 \mu \mathrm{m}$ (C and D); $40 \mu \mathrm{m}$ (A, inset, and B, top inset); $15 \mu \mathrm{m}$ (B, bottom inset, and D, insets). (E and F) Western blot analysis of ADK of total homogenates from control autopsy hippocampus and HS specimens. (E) Representative immunoblots. (F) Densitometric data, expressed relative to optical density of $\beta$-actin $\left(n=5\right.$ per group). Data are mean \pm SEM. ${ }^{*} P<0.05$ vs. control.

$\beta H B$ measurement. Mice were deeply anesthetized and blood collected via cardiac puncture into EDTA-containing syringes. After centrifugation, plasma was assayed for $\beta \mathrm{HB}$ with a Precision Xtra monitor and ketone test strips (Abbott Laboratories).

Human material. Human specimens were a gift from J.C. Baajen (University of Amsterdam Academic Medical Center and VU University Medical Center, Amsterdam, The Netherlands). Written informed consent was obtained from patients or their guardians, and brain tissue was handled in a manner compliant with the guidelines and approval of AMC's Medical Ethics Committee (MEC; University of Amsterdam, Amsterdam, The Netherlands). Patients underwent resection for refractory epilepsy and had predominantly medically intractable complex partial seizures. We examined 11 surgical epilepsy specimens: 6 with HS (mean age, 26.4 years; range, 18-42 years) and 5 without HS (mean age, 29.5 years; range, 18-41 years; with a focal lesion not involving hippocampus proper and no appreciable neuronal loss or reactive gliosis observed in hippocampus). Normal-appearing control hippocampus was obtained at autopsy from adults (mean age, 31 years; range, 18-35 years) without a history of seizures or other neurological diseases within 12 hours of death. Tissue was fixed, paraffin embedded, sectioned $(6 \mu \mathrm{m})$, and processed for hematoxylin and eosin and immunocytochemistry for GFAP (monoclonal mouse, 1:4,000; DAKO) and ADK (polyclonal rabbit, 1:500) (6). Single- and double-label immunocytochemistry was performed as described previously (16).

Western blotting. Freshly frozen histologically normal autopsy hippocampus and HS samples ( $n=5$ per group) were homogenized. $30 \mu$ g protein was separated by standard gel electrophoresis, transferred, and incubated overnight in buffer $(20 \mathrm{mM}$ Tris, $150 \mathrm{mM} \mathrm{NaCl}, 0.1 \%$ Tween, $5 \%$ nonfat dry milk, $\mathrm{pH} 7.5)$ containing the primary ADK antibody $(1: 5,000)$. Preparation and analysis of Western blots were performed as described previously (16). Mouse brain was processed similarly using $50 \mu \mathrm{g}$ protein derived from WT whole brain extracts $(n=4 ; \mathrm{CD}$ and $\mathrm{KD})$. ADK levels were normalized to internal standards and reported relative to control. Densitometry was performed using Image (NIH).

Statistics. Seizure data were analyzed using StatView for Windows (version 5.0.1; SAS Institute Inc.), reported as mean \pm SD from 5 hours of blinded recording from each animal and condition, and analyzed using 1-way ANOVA followed by Bonferroni/Dunn multiple test for individual means. Data for ADK and ketone quantification were reported as mean \pm SEM and analyzed using 2-tailed Student's $t$ test. A $P$ value of 0.05 or less was considered significant.

\section{Acknowledgments}

This study was supported by the NIH (NS058780, NS061844, NS065957, NS066932, 2P20RR00017699), National Science Foundation (IOS-0843585), Swedish Research Council, CHDI Foundation, National Epilepsy Fund (09-05), EU FP7 (NeuroGlia), Grant Agreement 202167, Trinity College, and the Legacy Foundations. We thank Cory Szybala and Jenny Nord for assistance with mouse breeding.

Received for publication March 2, 2011, and accepted in revised form April 20, 2011.

Address correspondence to: Detlev Boison, Legacy Research Institute, R.S. Dow Neurobiology Laboratories, 1225 NE 2nd Ave., Portland, Oregon 97232, USA. Phone: 503.232.0589; Fax: 503.232.5465; E-mail: dboison@downeurobiology.org. 
1. Kossoff EH, Rho JM. Ketogenic diets: evidence for short- and long-term efficacy. Neurotherapeutics. 2009;6(2):406-414.

2. Neal EG, et al. The ketogenic diet for the treatment of childhood epilepsy: a randomised controlled trial. Lancet Neurol. 2008;7(6):500-506.

3. Kawamura M Jr, Ruskin DN, Masino SA. Metabolic autocrine regulation of neurons involves cooperation among pannexin hemichannels, adenosine receptors, and $\mathrm{K}_{\mathrm{ATP}}$ channels. J Neurosci. 2010;30(11):3886-3895.

4. Dunwiddie TV, Worth T. Sedative and anticonvulsant effects of adenosine analogs in mouse and rat. J Pharmacol Exp Ther. 1982;220(1):70-76.

5. Etherington LA, Frenguelli BG. Endogenous adenosine modulates epileptiform activity in rat hippocampus in a receptor subtype-dependent man- ner. Eur J Neurosci. 2004;19(9):2539-2550.

6 . Li T, et al. Adenosine kinase is a target for the prediction and prevention of epileptogenesis in mice. J Clin Invest. 2008;118(2):571-582.

7. Pignataro G, Simon RP, Boison D. Transgenic overexpression of adenosine kinase aggravates cell death in ischemia. J Cereb Blood Flow Metab. 2007;27(1):1-5

8. Wilz A, Pritchard EM, Li T, Lan JQ, Kaplan DL, Boison D. Silk polymer-based adenosine release: Therapeutic potential for epilepsy. Biomaterials. 2008;29(26):3609-3616.

9. Johansson B, et al. Hyperalgesia, anxiety, and decreased hypoxic neuroprotection in mice lacking the adenosine $\mathrm{A}_{1}$ receptor. Proc Natl Acad Sci US A. 2001;98(16):9407-9412.

10. Boison D. The adenosine kinase hypothesis of epi- leptogenesis. Prog Neurobiol. 2008;84(3):249-262.

11. D'Ambrosio R, et al. Functional definition of seizure provides new insight into post-traumatic epileptogenesis. Brain. 2009;132(pt 10):2805-2821.

12. Eagles DA. Design of dietary treatment: humans versus rodents. Epilepsia. 2008;49 suppl 8:61-63.

13. Juge $\mathrm{N}$, et al. Metabolic control of vesicular glutamate transport and release. Neuron. 2010;68(1):99-112.

14. Ma W, Berg J, Yellen G. Ketogenic diet metabolites reduce firing in central neurons by opening $\mathrm{K}_{\mathrm{ATP}}$ channels. J Neurosci. 2007;27(14):3618-3625.

15. During MJ, Spencer DD. Adenosine: a potential mediator of seizure arrest and postictal refractoriness. Ann Neurol. 1992;32(5):618-624.

16. Aronica E, et al. Expression and localization of voltage dependent potassium channel Kv4.2 in epilepsy associated focal lesions. Neurobiol Dis. 2009;36(1):81-95. 